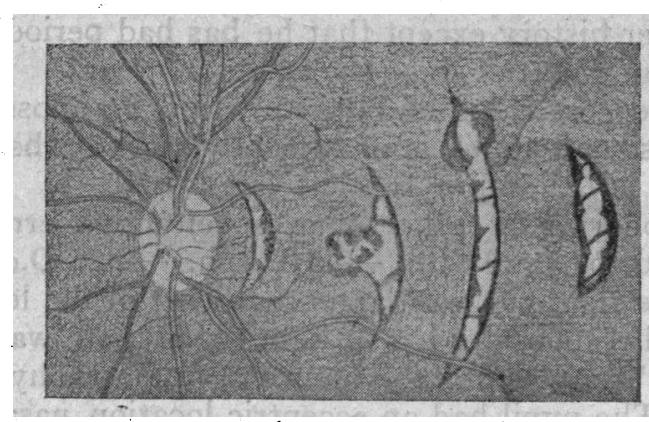

FIG. 2.

macula. : The second rupture which is shaped somewhat like the head of a spear seems to be quite close to the macula. The third and fourth ruptures are typical crescentic injuries.

'About the time I was preparing to report this case I saw a second case with four choroidal ruptures. The patient was a coloured soldier who injured his eye several years ago. His vision in this right eye is $8 / 200$. Since no fundus camera was available I attempted to make a fundus drawing of the condition which existed.

I think these two cases illustrate clearly why vision may be retained in certain cases of rupture through the region of the macula and almost completely lost in others. The second case illustrates the amount of haemorrhage and pigmentation which may occur with choroidal rupture through the macula and also how the distinct anatomy of the macula may modify the traumatic pathology. The first case is remarkable in that visual acuity of $20 / 20$ was obtained by correction, although the visual acuity changes with movement of the eye since the lens is not anchored.

\title{
THE RELATIONSHIP OF OPHTHALMOLOGY AND RHINOLOGY*
}

\section{BY}

\author{
V. E. NEGUS \\ LONDON
}

Dr. A. C. Reid in introducing the speaker referred to the difficulties of the average ophthalmologist when a case had an oto-rhinological aspect. For years he and others had been referring such cases from the hospital to King's. Now they would listen with interest to what Mr. Negus had to say.

* A report of a meeting of the Royal Eye Hospital Clinical Society held on September 24, 1943. 
Mr. V. E. Negus agreed that the two specialities had far too few opportunities for discussion and mutual criticism. He would begin by discussing the penetrating injuries and fractures of the orbit. If a penetrating injury involved maxillary antrum or ethmoid, he advised the removal of any loose fragments of bone, or foreign bodies. Sulphanilamide powder should be applied to such wounds. It must not be forgotten that there was close connection between the nasal sinuses and the cranial cavity; unnecessary interference might easily give rise to meningitis. Blood in sinuses usually absorbed. Gross injuries to the antrum were best approached through the sublabial route. Sometimes the second division of the 5th was involved in an injury; this did not call for interference unless neuralgia ensued, when he advocated alcohol injection of the nerve. In depressed fracture of the malar bone the bone should in the early stage of the injury be elevated by a Kilner's lever inserted through the temporal region. Prompt action could prevent a troublesome deformity.

The lacrimal gland did not often enter his sphere. He had noted cessation of lacrimation when the geniculate ganglion of the facial was affected by herpes. The secretory fibres ran through the great superficial petrosal and Vidian nerves to Meckel's ganglion and thence to the lacrimal gland. Sometimes ophthalmologists had asked him to inject Meckel's ganglion in cases of excessive lacrimation. He deprecated the habit of injecting the ganglion through the orbital fissure. It hurt the patient, involved danger to the optic nerve, and was more difficult than the lateral route. As compared with injection of the ganglion through the posterior palatine canal or sphenopalatine foramen, the lateral route through the pterygomaxillary fossa was easy and without serious danger. No case should be submitted to injection unless the application of cocaine paste behind the posterior end of the middle turbinal had previously shewn that lacrimation could be stopped by blocking the ganglion.

He now came to the subject of the lacrimal sac." This structure should not be forgotten when operating on frontal or ethmoidal sinuses through the external route. The usual incision did not involve the sac; but recently an operation had been introduced in which an incision below the orbit was utilised. In the best hands this route might give good results but it was fraught with danger to the nasal duct.

Chronic dacryocystitis was a condition met with by ophthal- . mologist and rhinologist alike. Excision of the sac might sometimes be necessary; but the re-constitution of the passages was a more physiological ideal. 'In the past West's operation of opening the sac through the nose was employed; but he thought the 
operation had now been superseded by the external operation of Toti, or better the modern modification of Dupuy-Dutemps. In the past he had maintained that these were operations for the rhinological surgeon; but since seeing some good results of $\mathrm{Mr}$. Savin's he would now only say that nobody should perform the operation unless he was prepared to take the trouble to acquire the special technique.

Mr. Negus regretted the misleading label of orbital cellulitis. These were really cases of frontal sinusitis and acute ethmoiditis with orbital oedema. Usually they should be treated by lavage of the maxillary sinus, which was always also infected and by an ephedrine spray. External incision was very rarely necessary or advisable. Some cases of orbital cellulitis in children were due to osteomyelitis of the maxilla or malar bone.

The minor procedure of antrum puncture could occasionally be dangerous if the cannula penetrated the orbit and air was injected. Care should be taken to inject some saline before blowing in air.

Cases of retrobulbar neuritis were referred to the rhinologist. There were many cases of ethmoidal and sphenoidal suppuration in which the optic nerve' was not affected. Conversely the cases of papilloedema and retrobulbar neuritis generally did not seem to be associated with nasal sepsis. In his experience the sphenoidal sinus was seldom infected in the absence of infection of the adjacent posterior ethmoidal air cells. Even though sepsis was absent, the local blood-letting might occasionally be advantageous.

He was often asked to look for focal sepsis in such cases as iridocyclitis of obscure origin. Often the tonsils were causative factors of infection, seldom if ever suppuration of the nasal sinuses.

He now came to the subject of neoplasms. One had occasionally to deal with osteomas of the orbit. These had a soft base and were not so difficult to deal with as the ivory exostoses of the external auditory meatus. Naso-pharyngeal fibromas sometimes went first to the ophthalmologist because of associated proptosis. They also had middle ear deafness, and were difficult to deal with because they might involve the carotid. Some were radio-sensitive. Rodent ulcer came into the sphere of both specialities. He wished to emphasise the danger of irradiating all such tumours. He had often seen intractable bone necrosis result; and some of these cases could have been dealt with by excision. Even if bone

- was involved wide excision followed by diathermy might give good results.

The eyes were sometimes displaced by a carcinoma of the antrum, or by mucocele of the frontal sinus or ethmoid. The former were usually best attacked by the sublabial rather than by the external route. 
Mr. Negus then discussed facial paralysis. He reminded ophthalmologists that obstinate cases might be treated by decompression, by nerve grafting, or by fascia lata slings.

$\mathrm{He}$ also discussed petrositis in which otorrhoea might be associated with an abscess at the tip of the petrous bone. In these cases pain behind the eye was a prominent symptom. If the 6 th nerve was involved by localised meningitis Gradenigo's syndrome resulted.

Allergy in the form of hay fever was of mutual interest. Desensitisation and medical treatment was sometimes ineffective; and zinc ionization of the nasal mucous membrane often helped. Curiously enough the conjunctivitis often improved concurrently.

Dr. Robertson said hay fever was indeed an odd disease. He had known a case where the nose cleared up after' local treatment to the eyes and dark glasses. It was probably due to the absence of the exciting atopen. One must beware of confusing post hoc with propter hoc.

Mr. A. D. Griffith said he did not remember when he had enjoyed a lecture more than that just delivered by $\mathrm{Mr}$. Negus. He entirely agreed in condemning the external incision of so-called orbital cellulitis. He had only once incised such a case, and he had then regretted it. He was a little surprised at what Mr. Negus had said about the operation of dacryo-cystorrhinostomy and its dangers. It was an operation demanding care; but he had never found himself in any particularly difficult situation. 'He thought radium good treatment for rodents; but of course one should employ care as to dosage and not be afraid of obtaining skilled technical advice from a physicist. Bone necrosis should never occur with proper dosage. The ophthalmologist could help in the insertion of radon by the orbital route in naso-pharyngeal fibro-sarcoma.

Mr. L. H. Savin said he liked doing dacryo-cystorrhinostomies, and Mr. Negus had spoken kindly about his results; but after an excursion into an ethmoid he had often thought it an operation more satisfying to the surgeon than advantageous to the patient. A rhinologist would do the job better. Viewed objectively he thought the job was really one for collaboration between rhinologist and ophthalmologist. Certainly expert opinion should always be obtained on the nose and the state of its mucous membrane before operating.

Recently he injected the second division of the fifth through the inferior orbital fissure. He was asked to do it for inoperable carcinoma of the antrum. He had not thought he was endangering the optic nerve. He was interested to hear that the mastoid should always be explored in petrositis. He remembered sending such a case to a rhinologist who adopted conservative treatment. Three days later the patient died suddenly from meningeal infection. The case had come to the eye department because of associated 6th nerve paralysis. He hoped Mr. Negus would say a few words about vacuum headache.

In reply Mr. Negus again stressed that the lateral route was the easiest in the injection of Meckel's ganglion. Gradenigo's syndrome differed from petrositis. Vacuum headache was a very real thing. The pain occurred in the morning and was confined to the region of one frontal sinus. When the pain was relieved the patient might hear the small hiss of the entering air. There were almost always rhinological signs of obstruction to the opening of the sinus. Sometimes an operation for deviation of the nasal septum would cure the condition.

Before Mr. Negus's paper Dr. Holmes-Smith shewed a case of Sjogren's syndrome of kerato-conjunctivitis sicca. This was a condition more common in Scandinavia in which polyarthritis was associated with lack of lacrimal secretion, though there was still abundant mucous conjunctival secretion. In late cases the eye condition simulated essential shrinkage of the conjunctiva. Sometimes occluding the puncta was said to help by preventing. drainage of such tears as were present. This measure had not proved nceessary in the case shewn which had responded to vitamin therapy to some extent. 